\title{
Laser-triggered proton acceleration from hydrogenated low-density targets
}

\author{
A. V. Brantov, ${ }^{1,2}$ E. A. Obraztsova, ${ }^{3,4}$ A. L. Chuvilin, ${ }^{5,6}$ \\ E. D. Obraztsova, ${ }^{4}$ and V. Yu. Bychenkov ${ }^{1,2}$ \\ ${ }^{1}$ P. N. Lebedev Physics Institute, Russian Academy of Science, Leninskii Prospect 53, \\ Moscow 119991, Russia \\ ${ }^{2}$ Center for Fundamental and Applied Research, Dukhov Research Institute of Automatics (VNIIA), \\ Moscow 127055, Russia \\ ${ }^{3}$ Shemyakin and Ovchinnikov Institute of Bioorganic Chemistry, RAS, Moscow, 117997 Russia \\ ${ }^{4}$ A. M. Prokhorov General Physics Institute, Russian Academy of Science, Vavilov Str. 38, \\ Moscow 119991, Russia \\ ${ }^{5}$ CIC nanoGUNE Consolider, Tolosa Hiribidea 76, E-20018 San Sebastian, Spain \\ ${ }^{6}$ KERBASQUE, Basque Foundation for Science, Maria Diaz de Haro 3, 48013 Bilbao, Spain
}

(Received 5 October 2016; published 19 June 2017)

\begin{abstract}
Synchronized proton acceleration by ultraintense slow light (SASL) in low-density targets has been studied in application to fabricated carbon nanotube films. Proton acceleration from low-density plasma films irradiated by a linearly polarized femtosecond laser pulse of ultrarelativistic intensity was considered as result of both target surface natural contamination by hydrocarbons and artificial volumetric doping of low-density carbon nanotube films. The 3D particle-in-cell simulations confirm the SASL concept [A. V. Brantov et al., Synchronized Ion Acceleration by Ultraintense Slow Light, Phys. Rev. Lett. 116, 085004 (2016)] for proton acceleration by a femtosecond petawatt-class laser pulse from realistic lowdensity targets with a hydrogen impurity, quantify the characteristics of the accelerated protons, and demonstrate a significant increase of their energy compared with the proton energy generated from contaminated ultrathin solid dense foils.
\end{abstract}

DOI: 10.1103/PhysRevAccelBeams.20.061301

\section{INTRODUCTION}

Laser-driven proton acceleration is a topic of extraordinary interest for fundamental research and possible applications in nuclear physics [1], laboratory astrophysics [2], inertial confinement fusion [3], proton radiography [4], nuclear medicine and biology [5], and extreme states of matter [6]. These issues motivated a worldwide search for different ion acceleration mechanisms [7] with the aim to maximize both the yield and the energy of the protons. In this context, an important role is played by low-density targets with an electron density close to the relativistic critical density. In the last few years, these targets have already shown promising results for effective ion acceleration.

The most widely accepted basic idea for using near-critical planar targets is to increase laser absorption by the target thus enhancing target normal sheath acceleration (TNSA) $[8,9]$ or ion acceleration through affective volumetric heating $[10,11]$. One more approach is to use the near-critical layer for relativistic pulse shaping and fast focusing to reach the radiation pressure acceleration (RPA) regime [12].

Published by the American Physical Society under the terms of the Creative Commons Attribution 4.0 International license. Further distribution of this work must maintain attribution to the author(s) and the published article's title, journal citation, and DOI.
A principally new concept for using low-density targets for proton acceleration was recently proposed based on the synchronized propagation of the laser pulse and proton bunch [13]. The key points of this acceleration mechanism are to stop the laser pulse at the front of the target and then accelerate the infiltrating intense part of the pulse inside the plasma at the same rate as the proton energy increase in the ponderomotive potential to achieve synchronized acceleration by slow light (SASL). In this concept, the linearly polarized laser pulse propagates and increases its group velocity as a result of increasing plasma transparency as the ponderomotively driven electron spike on the down-going pulse ramp disappears during pulse propagation.

The 3D particle-in-cell (PIC) simulations in support of the SASL mechanism were performed with the example of a low-density $\mathrm{CH}_{2}$ target [13]. Such a material does not yet exist, and a suitable hydrogenated material is highly desirable as a substitute from the experimental standpoint. For applications, it is required to produce low-density films homogeneous at the $\mu \mathrm{m}$-scale with a density one to two orders of magnitude below the solid state density and with reasonable flexibility to tailor target parameters. These requirements make fabricating the target a fundamental research and advanced technology issue in the field of material nanoscience and technology $[14,15]$. Nanostructured targets are also discussed as a way of increasing the efficiency of lasertarget coupling and proton acceleration [16,17]. 

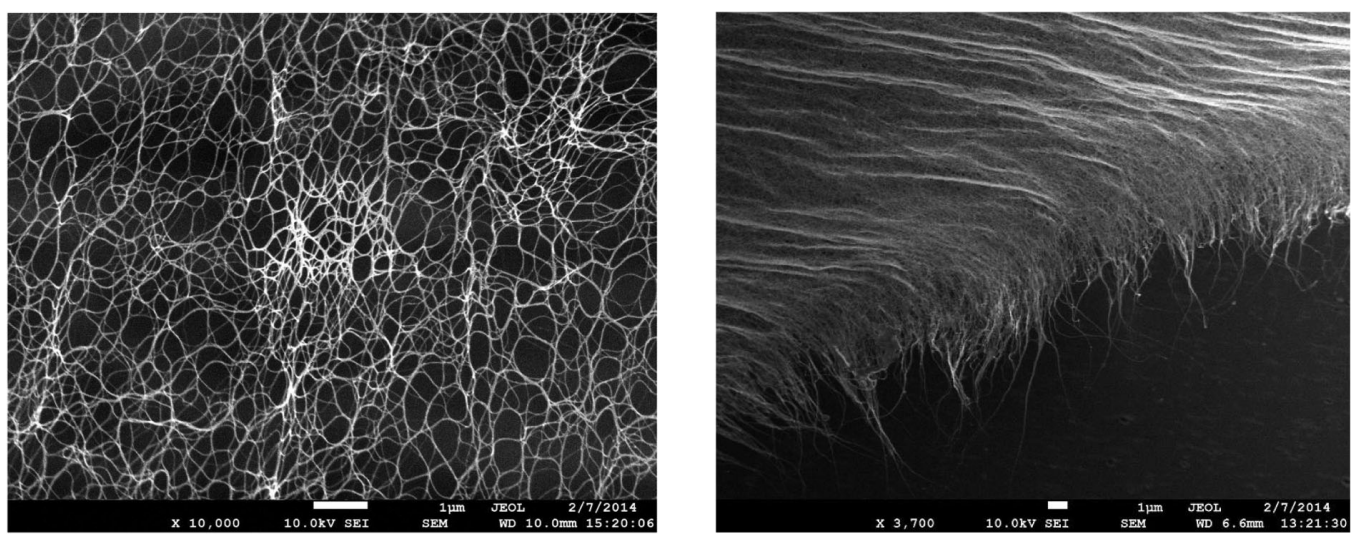

FIG. 1. The SEM images for the SWNT films with a density of $0.1 \mathrm{mg} / \mathrm{cm}^{3}$ (left panel) and $1 \mathrm{mg} / \mathrm{cm}^{3}$ (right panel) synthesized by an aerosol method with different deposition times.

In this paper, we extend recently published results of SASL simulations [13] with hypothetical low-density targets to targets that are available in practice. In Sec. II, we describe low-density carbon nanotube films produced in the Prokhorov General Physics Institute of the Russian Academy of Sciences (GPI RAS). In Sec. III, we present 3D PIC simulations of proton acceleration from low-density carbon nanotube film with hydrocarbon contaminated surfaces. In Sec. IV, we present similar simulations for volumetrically hydrogenated low-density carbon targets. Section V contains our conclusions.

\section{FABRICATION OF LOW-DENSITY FILMS ON A BASE OF CARBON NANOTUBES}

Two approaches are now available in the GPI RAS for producing films of assembled single-wall carbon nanotubes (SWNTs). The SWNTs with an average diameter of 1.6-2.2 nm were synthesized by an aerosol technique [18]. The films were deposited onto nitrocellulose filter on the bottom of the synthesis chamber. The nitrocellulose filter has a very weak adhesion, which allows reprinting the SWNT film onto any desirable substrate. Both the film density and its thickness can be varied by changing the deposition time. The SWNT films were characterized using scanning electron microscopy (SEM). In Fig. 1, the SEM images of the SWNT films with densities of $0.1 \mathrm{mg} / \mathrm{cm}^{3}$ (left) and $1 \mathrm{mg} / \mathrm{cm}^{3}$ (right) are shown. The typical thicknesses of the SWNT films produced by the aerosol technique is $100-200 \mathrm{~nm}$. Thicker films can be produced by increasing the deposition time. For example, a $1 \mathrm{mg} / \mathrm{cm}^{3} 30 \mu \mathrm{m}$ SWNT foil can be produced during a deposition time of a few hours.

The thicker SWNT targets (see Fig. 2) were formed from SWNTs with an average diameter of $1.4 \mathrm{~nm}$ synthesized by an electrical arc discharge using a Ni/ $\mathrm{Y}_{2} \mathrm{O}_{3}$ catalyst in an Ar atmosphere [19]. The SWNT targets with a density of $30-50 \mathrm{mg} / \mathrm{cm}^{3}$ required for the experiments with relativistically intense laser pulses were deposited on quartz or $\mathrm{Al}_{2} \mathrm{O}_{3}$ substrates. The SWNT films with a thickness of
$5-10 \mu \mathrm{m}$ are routinely produced by this technique by decanting through the filter an aqueous suspension containing $8-10 \mathrm{mg}$ of a raw arc synthesis soot, containing about $20 \%$ of single-wall carbon nanotubes. We expect that the technique used allows us to fabricate thicker films, e.g., the twice-thicker film will require about $20 \mathrm{mg}$ of soot.

To enrich the films with hydrogen, the nanotubes were filled with coronene $\left(\mathrm{C}_{24} \mathrm{H}_{12}\right)$ molecules [20]. Prior to the filling procedure, the nanotube caps were opened by heating in air for $25 \mathrm{~min}$ at $450{ }^{\circ} \mathrm{C}$. The filling was performed in sealed glass tubes using the gas phase technique. The SWNTs in the glass tubes were spatially separated from the coronene powder (Angene). The treatment was performed in an $\mathrm{Ar}$ atmosphere at $450{ }^{\circ} \mathrm{C}$ for $13 \mathrm{~h}$. After synthesis, samples were washed in toluene to remove coronene and side products from the outer surface of the nanotubes. Additional heating was performed for $2 \mathrm{~h}$ in an $\mathrm{Ar}$ atmosphere in glass tubes without coronene powder. The structural characterization of such targets

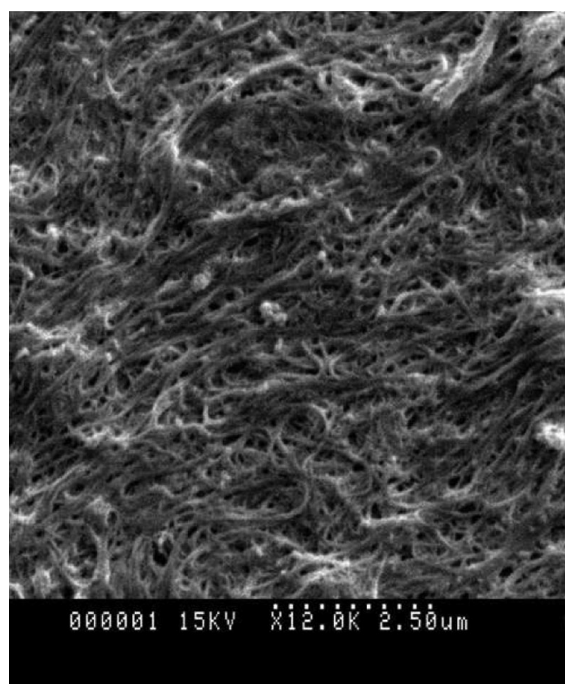

FIG. 2. The SEM image of the film formed from SWNTs synthesized by an arc discharge technique. 

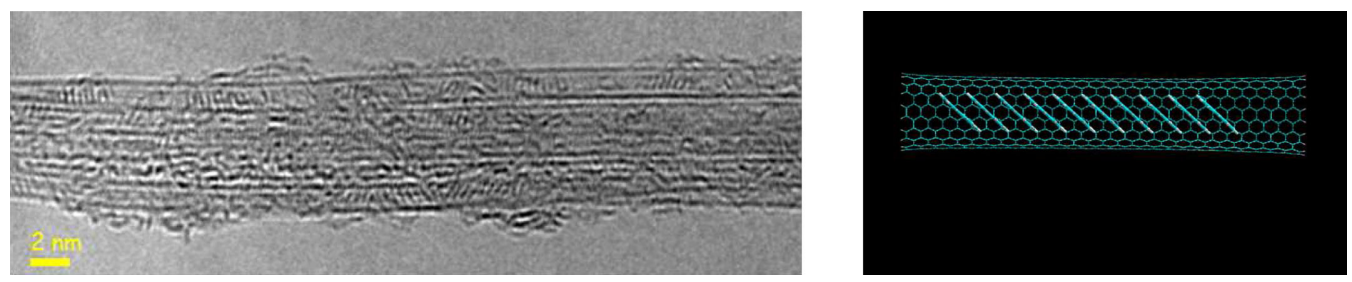

FIG. 3. The HRTEM image of coronene $\left(\mathrm{C}_{24} \mathrm{H}_{12}\right)$ stacks inside SWNTs (left panel) and a computer modeling [21] of stacking (right panel).

with high-resolution transmission electron microscopy (HRTEM) shows that coronene molecules are stacked inside the nanotubes (Fig. 3). With this technique, the mass fraction of hydrogen in the SWNT-coronene target reaches $2 \%$.

\section{3D PIC SIMULATIONS OF PROTON ACCELERATION FROM A LOW-DENSITY FILM WITH HYDROCARBON CONTAMINATED SURFACES}

In experiments, a surface of porous carbon nanotube film is naturally contaminated by hydrocarbons and can therefore be used for laser-triggered proton acceleration. In this section, we present a numerical simulation of laser acceleration of protons from surface-contaminated thin solid foils and low-density plasma targets using the 3D PIC code MANDOR [22]. A linearly polarized pulse of $30 \mathrm{~J}$ energy with a maximum intensity $5 \times 10^{21} \mathrm{~W} / \mathrm{cm}^{2}$ (a dimensionless laser field amplitude $a_{0} \approx 60$ for the wavelength $\lambda=1 \mu \mathrm{m}$ ) is focused on the front side of a target at normal incidence. It has a Gaussian shape in time (FWHM duration is $\tau=30 \mathrm{fs}$ ) and in the transverse direction (FWHM focal spot size is $D_{f}=4 \mu \mathrm{m}$ ). In the numerical PIC method we model carbon nanotube substrate as a plasma with homogeneous electron density equal to the averaged electron density of fully ionized carbon in the target of given mass density. This is a quite adequate model for relativistically strong laser field and extremely small characteristic space scales of the nanostructured material. The typical diameter of carbon nanotubes is $1.2 \mathrm{~nm}$, the distances between them are $10-100 \mathrm{~nm}$ with characteristic scale $\sim 30 \mathrm{~nm}$ (porous size). The Coulomb field of such ionized nanotube may confine electrons only for weak laser field, $a_{0}<1$. The quiver distance of the oscillating electrons can be less than the porous size for the same condition, $a_{0}<1$. These estimates confirm that for ultrarelativistic intensities (considered here) electrons from different nanotubes are well mixed in the laser channel and laser pulse propagates within homogenized electron fluid. As concerning the small scale ion skeletons, it is even not important whether or not they well smoothed due to Coulomb explosion since they do not affect laser pulse propagation in a target. As targets, we assume a low-density carbon nanotube film with electron density $n_{e}=20 n_{c}$ (case 1 ) and an ultrathin solid carbon foil $\left(n_{e}=200 n_{c}\right.$, case 2$)$ of optimum thicknesses (respectively $6 \lambda$ and $0.12 \lambda$ ) with two contamination surface layers, one on each side $\left(n_{e}=n_{p}=20 n_{c}\right)$. Here $n_{c}$ is the plasma critical density, $n_{p}$ is the proton density. The thickness of the contamination layers is $\delta=20 \mathrm{~nm}$. As an alternative in the case of a low-density carbon target (case 3), we also use a proton impurity distributed near a surface inside the target with a linearly decreasing density profile such that the proton density changes from $4 n_{c}$ at the target boundary to zero at the distance $0.2 \lambda$, which gives the same number of protons as in the first case. We also perform a run (case 4) for a proton contamination layer of thickness $0.2 \lambda$ with a homogeneous proton density of $4 n_{c}$, which doubles the total proton number compared with case 3 .

In our 3D simulations, we used a numerical mesh with a cell size $x \times y \times z=0.01 \lambda \times 0.05 \lambda \times 0.05 \lambda$. The pulse, linearly polarized in the $z$ direction, propagates in the $x$-direction, from the left border of the simulation domain to the right. A number of runs have been performed with the longitudinal resolution twice as fine $(\lambda / 200)$, aiming to better resolve a skin-depth length in the case of thin solid-density foils. The size of the simulation domain was 20-60 $\lambda$ in the $x$ direction and $20 \lambda$ transversely (cf. Fig. 4, bottom). The target, placed at a distance of $3-5 \lambda$ from the left boundary of the domain, transversely spanned the entire domain (as can be seen in Fig. 4, bottom). Each simulation ran for 300 fs. For the solid-density foils, we used 12 macroparticles (first order particles) of each species per cell, with the total number of particles of about $2 \times 10^{8}$. For the thick low-density targets, we used 2 particles of each species per cell inside the main target and 6 particles of each species per cell for electrons and protons inside the contamination layer (the total number of particles close to $10^{9}$ ).

The proton phase space as well as proton space distribution in Fig. 4 show proton acceleration from a low-density target in the SASL regime (case 1). Most energetic protons are accelerated from the front side of the target. They gain the most energy inside the target (compare $t=97 \mathrm{fs}$ and $t=195 \mathrm{fs}$ in Fig. 4). The conventional back side TNSA is less effective in this case. This can be clearly seen by comparing the velocities of the protons generated at the front and rear sides of the target for all times. We compared the efficiency of SASL with proton acceleration from a solid carbon foil of optimum thickness with surface hydrogen contamination that gives the maximum proton energy [23]. The latter case corresponds to volumetric 

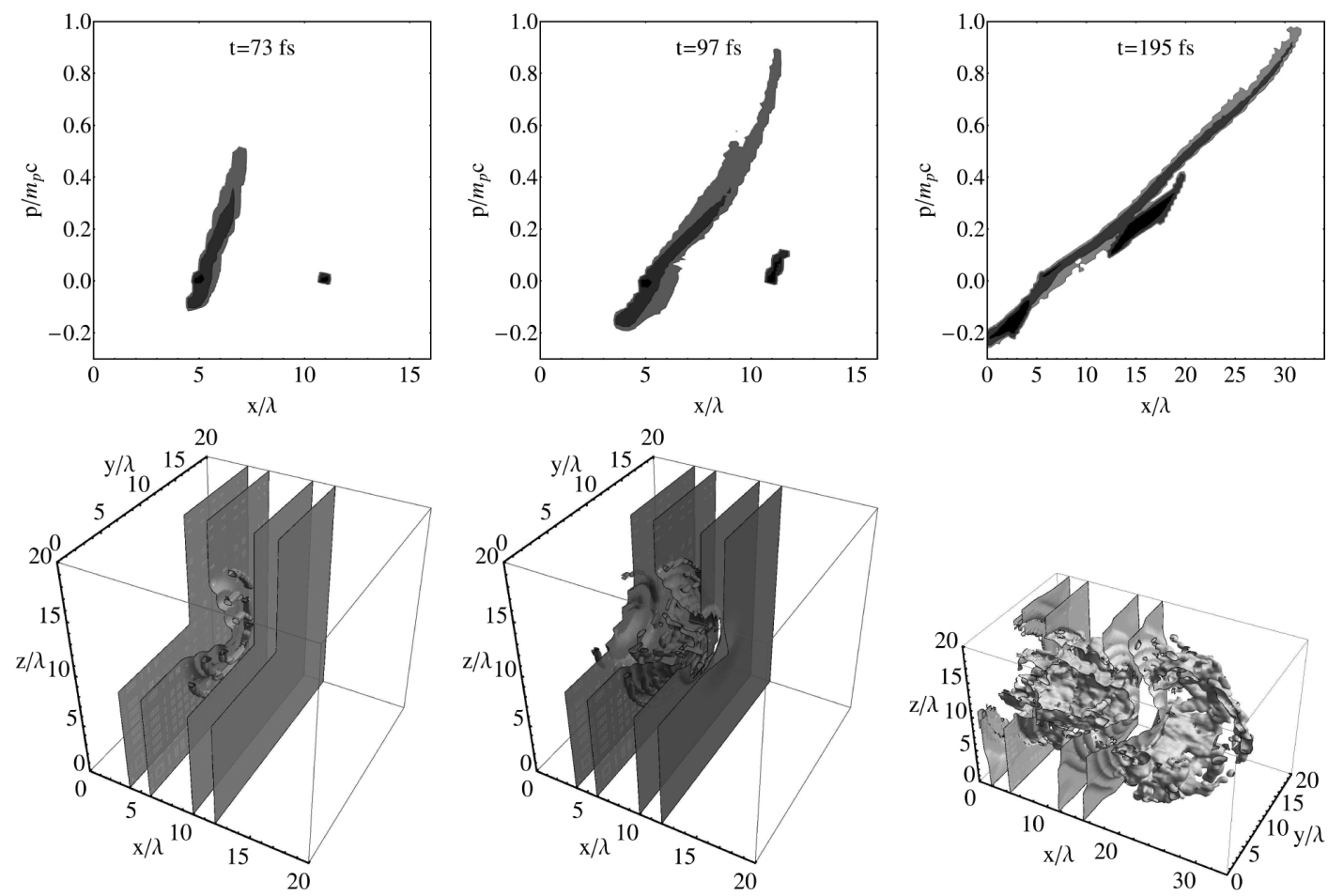

FIG. 4. Phase space (top) and density counter plot (bottom) of protons accelerated from contamination layers of low-density carbon target (case 1) at three instants: the film is situated between $x=5 \lambda$ and $x=11 \lambda$.

target heating $[10,23,24]$ where several mechanisms of proton acceleration work all together: Coulomb explosion, TNSA, and radiation pressure. The spectra of protons from the targets considered are shown in Fig. 5. The SASL regime gives a twofold energy increase compared with the case of proton acceleration from a thin solid foil of optimum thickness (compare the black and gray lines in Fig. 5). This is similar to what was demonstrated for hypothetical $\mathrm{CH}_{2}$ targets [13]. We also studied the role of a more deeply distributed hydrogen contamination (gray dashed curve in Fig. 5) and found that the deeper penetration of the proton impurity inside the target has little effect on the maximum proton energy. At the same

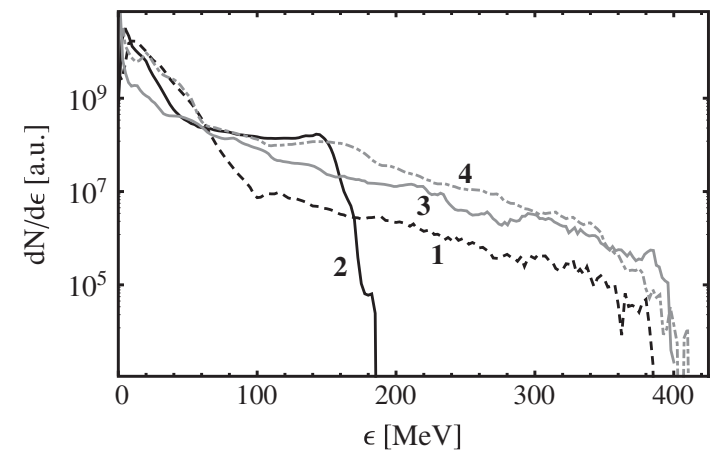

FIG. 5. Spectra of protons accelerated from the contamination layer of a solid foil (black curve, case 2) and a low-density target in cases 1,3 , and 4 (the respective dashed black, solid gray, and dash-dotted gray curves). time, the deeper penetration of the proton impurity increases the number of high-energy protons. Because of the smooth redistribution of the same number of protons near the front target boundary, more protons became involved in the SASL regime. In addition, the total number of high-energy particles increases with the average proton density inside the target contamination layer (compare the solid and dashed gray curves corresponding to cases 3 and 4). The peak ponderomotive potential inside the target, $\sim 65 \mathrm{MeV}$ [13], would be the energy that an ion gains in passing through the electrical sheath. Conversely, in the SASL case, the protons move together with the sheath, which increases the acceleration distance multifold. We have estimated the number of protons with the energy in excess of ponderomotive potential, $65 \mathrm{MeV}$, for the case 1 as $2 \times 10^{9}$ (bunch energy $\sim 0.03 \mathrm{~J}$ ) and for the case 4 as $1.5 \times 10^{10}$ (bunch energy $\sim 0.3 \mathrm{~J}$ ). For example, in the case 4 a bunch of these energetic protons contains approximately $30 \%$ of the total energy of all accelerated protons. The protons with the energy above $65 \mathrm{MeV}$ propagate from a target back side as a bunch with initial length of $10-\mu \mathrm{m}$ having a 20 degree divergence angle.

\section{3D PIC SIMULATIONS OF PROTON ACCELERATION FROM VOLUMETRICALLY HYDROGENATED LOW-DENSITY FILM}

Regarding the low-density carbon nanotube targets volumetrically enriched with hydrogen described in 


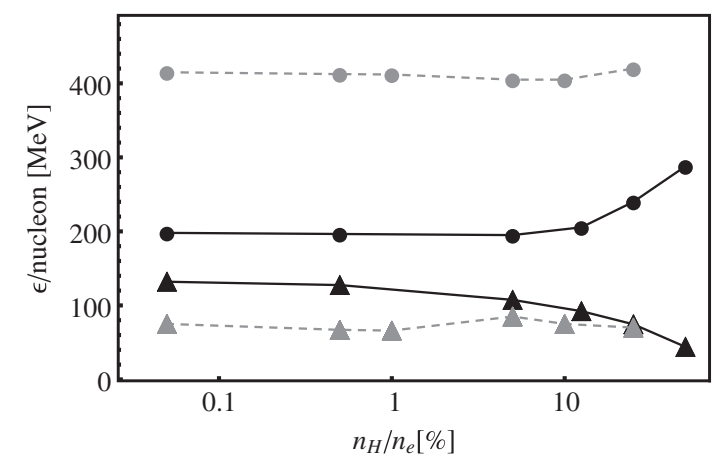

FIG. 6. Maximum proton (dots) and carbon ion (triangles) energies versus hydrogen concentration for the target electron density $200 n_{c}$ and thickness $l=0.12 \lambda$ (black curves) and for low-density targets with $n_{e}=20 n_{c}$ and $l=6 \lambda$ (gray dashed curves).

Sec. II, we here study the effect of a partial proton density on the maximum energy of laser accelerated ions.

To have a comparable example, we first simulated ion acceleration from hydrogenated solid-density targets of optimum thickness to ensure the maximum possible ion energy from such targets [10]. We used fully ionized solid-density carbon foils $\left(n_{e}=200 n_{c}=n_{H}+6 n_{C}\right)$ with different proton percentage contents. The optimum thickness of the target is $l=0.12 \lambda$. The maximum energies of protons (black dots) and $\mathrm{C}^{+6}$ ions (black triangles) for this target thickness are shown in Fig. 6. The maximum energy of protons accelerated from a thin solid foil of optimum thickness increases with the hydrogen concentration in accordance with the theory of plasma expansion into a vacuum or the TNSA mechanism [25]. It reaches the maximum value of $\sim 290 \mathrm{MeV}$ for a target with a hydrogen concentration $\sim 50 \%$. We note that a cryogenic hydrogen target gives practically the same proton energy (not shown). For carbon foils with a hydrogen impurity, the maximum proton energy is independent of the hydrogen concentration. The same energy $\simeq 200 \mathrm{MeV}$ is obtained for $0.05 \%, 0.5 \%$, and $5 \%$ hydrogen because the protons can be treated as test particles in a carbon environment. Correspondingly, the carbon ions also gain the same energy $\simeq 120 \mathrm{MeV}$ until hydrogen concentration exceeds 5\%, after which the carbon ion energy decreases.

We performed the same simulations with volumetrically hydrogenated low-density targets $\left(n_{e}=20 n_{c}\right)$. In contrast to the case of solid-density foils, we found no significant dependence of both protons and carbon ions on the hydrogen density for a target thickness $l \gtrsim 6 \lambda$. Such robustness is useful for controllable ion energies in experiments in which the availability of hydrogen concentration data is poor. The number of accelerated protons linearly increases with hydrogen density. For example, 3\% of hydrogen inside a target results in acceleration of $\sim 10^{9}$ protons with the energy in excess of $200 \mathrm{MeV}$.

\section{CONCLUSION}

As presented here, modern techniques for producing films of assembled single-wall carbon nanotubes (SWNTs), including hydrogen doping, with desirable mass densities and thicknesses open ways to implement them for lasertriggered ion generation. Such SWNT films are well suited for the recently proposed synchronized acceleration of protons by slow light (SASL) [13]. Based on 3D PIC simulation, we have shown the extraordinary efficiency of proton acceleration in terms of maximum proton energy from low-density carbon nanotube targets with hydrogen as a surface contamination and as a volumetric impurity. The energy conversion efficiency to the protons with energy in excess of laser ponderomotive potential increases almost proportionally to the partial hydrogen density until the latter stays considerably less than a carbon density and reaches about $1 \%$. This open a way for further increase of energy conversion efficiency with SASL if new low-density strongly hydrogenized targets could be produced. We hope that presented results will motivate future experimental works to confirm the SASL concept, especially because low-density SWNT targets are currently available.

\section{ACKNOWLEDGMENTS}

This work was supported by the Russian Science Foundation (Grant No. 17-12-01283) except the part devoted to the SWNT-coronene substance production and characterization, which was supported by the Russian Foundation for Basic Research (Grants No. 1532-20941, No. 15-32-70014).

[1] V. Yu. Bychenkov, V. T. Tikhonchuk, and S. V. Tolokonnikov, Nuclear reactions triggered by laseraccelerated high-energy ions, JETP 88, 1137 (1999).

[2] B. A. Remington, R. P. Drake, H. Takabe, and D. Arnett, A review of astrophysics experiments on intense lasers, Phys. Plasmas 7, 1641 (2000).

[3] V. Yu. Bychenkov, W. Rozmus, A. Maksimchuk, D. Umstadter, and C. E. Capjack, Fast ignitor concept with light ions, Plasma Phys. Rep. 27, 1017 (2001).

[4] M. Borghesi, D. H. Campbell, A. Schiavi, M. G Haines, O. Willi, A. J. MacKinnon, P. Patel, L. A. Gizzi, M. Galimberti, and R. J Clarke, Electric field detection in laser-plasma interaction experiments via the proton imaging technique, Phys. Plasmas 9, 2214 (2002).

[5] S. V. Bulanov and V. S. Khoroshkov, Feasibility of using laser ion accelerators in proton therapy, Plasma Phys. Rep. 28, 453 (2002).

[6] P. K. Patel, A. J. Mackinnon, M. H. Key, T. E. Cowan, M. E. Foord, M. Allen, D. F. Price, H. Ruhl, P. T. Springer, and R. Stephens, Isochoric Heating of Solid-Density Matter with an Ultrafast Proton Beam, Phys. Rev. Lett. 91, 125004 (2003). 
[7] A. Macchi, M. Borghesi, and M. Passoni, Ion acceleration by superintense laser-plasma interaction, Rev. Mod. Phys. 85, 751 (2013).

[8] M. Passoni, A. Zani, A. Sgattoni, D. Dellasega, A Macchi, I. Prencipe, V. Floquet, P. Martin, T. V. Liseykina, and T. Ceccotti, Energetic ions at moderate laser intensities using foam-based multi-layered targets, Plasma Phys. Controlled Fusion 56, 045001 (2014).

[9] I. Prencipe, A. Sgattoni, D. Dellasega, L. Fedeli, L. Cialfi, Il. Woo Choi, I. Jong Kim, K. A. Janulewicz, K. F. Kakolee, Hwang Woon Lee, Jae Hee Sung, Seong Ku Lee, Chang Hee Nam, and M. Passoni, Development of foam-based layered targets for laser-driven ion beam production, Plasma Phys. Controlled Fusion 58, 034019 (2016).

[10] A. V. Brantov, E. A. Govras, and V. Yu. Bychenkov, Ion energy scaling under optimum conditions of laser plasma acceleration from solid density targets, Phys. Rev. ST Accel. Beams 18, 021301 (2015).

[11] V. Yu. Bychenkov, A. V. Brantov, and E. A. Govras, Comparative study of ion acceleration by linearly polarized laser pulses from optimized targets of solid and nearcritical density, Plasma Phys. Controlled Fusion 58, 034022 (2016).

[12] J. H. Bin, W. J. Ma, H. Y. Wang, M. J. V. Streeter, C. Kreuzer, D. Kiefer, M. Yeung, S. Cousens, P. S. Foster, B. Dromey et al., Ion Acceleration Using Relativistic Pulse Shaping in Near-Critical-Density Plasmas, Phys. Rev. Lett. 115, 064801 (2015).

[13] A. V. Brantov, E. A. Govras, V. F. Kovalev, and V. Yu. Bychenkov, Synchronized Ion Acceleration by Ultraintense Slow Light, Phys. Rev. Lett. 116, 085004 (2016).

[14] H. Gu and T. M. Swager, Fabrication of free-standing, conductive, and transparent carbon nanotube films, Adv. Mater. 20, 4433 (2008).

[15] A. Zani, D. Dellasega, V. Russo, and M. Passoni, Ultra-low density carbon foams produced by pulsed laser deposition, Carbon 56, 358 (2013).

[16] J. Yu, W. Zhou, X. Jin, L. Cao, Z. Zhao, W. Hong, B. Li, and $\mathrm{Y}$. Gu, Improvement of proton energy in high-intensity laser-nanobrush target interactions, Laser Part. Beams 30, 307 (2012).
[17] A. Andreev, K. Platonov, J. Braenzel, A. Lübcke, S. Das, H. Messaoudi, R. Grunwald, C. Gray, E. McGlynn, and M. Schnürer, Relativistic laser nano-plasmonics for effective fast particle production, Plasma Phys. Controlled Fusion 58, 014038 (2016).

[18] A. Moisala, A. G. Nasibulin, D. P. Brown, H. Jiang, L. Khriachtchev, and E. I. Kauppinen, Single-walled carbon nanotube synthesis using ferrocene and iron pentacarbonyl in a laminar flow reactor, Chem. Eng. Sci. 61, 4393 (2006).

[19] V. L. Kuznetsov, A. N. Usoltseva, A. L. Chuvilin, E. D. Obraztsova, and J.-M. Bonard, Thermodynamic analysis of nucleation of carbon deposits on metal particles and its implications for the growth of carbon nanotubes, Phys. Rev. B 64, 235401 (2001).

[20] A. I. Chernov, P. V. Fedotov, A. V. Talyzin, I. V. Anoshkin, A. G. Nasibulin, E. I. Kauppinen, and E. D. Obraztsova, Optical properties of graphene nanoribbons encapsulated in single-walled carbon nanotubes, ACS Nano 7, 6346 (2013).

[21] A. I. Chernov, P. V. Fedotov, I. V. Anoshkin, A. G. Nasibulin, E. I. Kauppinen, V. L. Kuznetsov, and E. D. Obraztsova, Single-walled carbon nanotubes as a template for coronene stack formation, Phys. Status Solidi B 251, 2372 (2014).

[22] D. V. Romanov, V. Yu. Bychenkov, W. Rozmus, C. E. Capjack, and R. Fedosejevs, Self-Organization of a Plasma due to 3D Evolution of the Weibel Instability, Phys. Rev. Lett. 93, 215004 (2004).

[23] T. Esirkepov, M. Yamagiwa, and T. Tajima, Laser IonAcceleration Scaling Laws Seen in Multiparametric Particle-in-Cell Simulations, Phys. Rev. Lett. 96, 105001 (2006).

[24] A. Macchi, A. Sgattoni, S. Sinigardi, M. Borghesi, and M. Passoni, Advanced strategies for ion acceleration using highpower lasers, Plasma Phys. Controlled Fusion 55, 124020 (2013).

[25] A. P. L Robinson, A. R. Bell, and R. J. Kingham, Effect of Target Composition on Proton Energy Spectra in Ultraintense Laser-Solid Interactions, Phys. Rev. Lett. 96, 035005 (2006). 